\section{NP27 (continued)}

Evaluation: Preliminary results will be ready for distribution in Fall 2018.

Conclusions and Implications: Because childcare centers are policy-sensitive institutions, evidence underscoring the benefits of fruit and vegetable gardening may encourage regulators to adopt supportive rules (Tandon, Walters et al. 2016). With approximately $76 \%$ of the U.S. population living in areas with an annual growing season >200 days (IIASA 2013), a gardening component may be a promising obesity prevention strategy for young children in those regions, where $77 \%$ of total $(120,000$ approximatley) U.S. regulated childcare centers are located (CCAA 2012).

Funding: 2017-68001-26354.

\section{NP28 Peer-Led Text Message Intervention Effect on Fruit, Vegetable, and Beverage Intake Among Rural Adolescents}

Alison Gustafson, PhD, RD, jilcotts@ecu.edu, University of Kentucky, 206G Funkhouser, Lexington, KY 40506; Stephanie Jilcott Pitts, PhD, East Carolina University

Objective: There has been increased attention at the role that social media and technology may have in improving health outcomes. However, there remains limited interventions utilizing a peer based model with text messages as a way to improve dietary intake and decrease sugar-sweetened beverage intake. The goal of this study was to test a randomized control trial 8-week peer-led text message intervention among 14-16 year rural adolescents.

Description: Eight schools participated in the randomized control trial $(n=4$ intervention schools and $n=4$ control schools and $\mathrm{n}=350$ intervention students and $\mathrm{n}=150$ control). The text message consisted of primarily affective messages with a challenge each week related to consuming fruits, vegetables, or low-calorie beverages. Undergraduate dietetics and human nutrition students sent text messages on Tuesday and Saturday over the 8-week period via the "Group Me" app.

Evaluation: Intent to treat (ITT) analyses was conducted among all those that completed baseline and post intervention survey data $(n=339)$ compared to controls $(\mathrm{n}=151)$. A 3-way comparison was conducted as well among responders compared to non-responders and controls. Among those that participated in the intervention there was a .25 serving increase of fruit per week and a .34 serving increase of vegetables per week compared to control adolescents. Among those that responded frequently (more than two return text messages) there was a .75 serving increase of fruit per week and a .82 serving increase of vegetables per week compared to those that infrequently responded (less than two returned text messages).

Conclusions and Implications: A peer-led text message intervention appears to be effective at improving fruit and vegetable intake among rural adolescents. High level of engagement in texting between peers is associated with even greater gains in healthy food choices. Insurance companies, health departments, and other health care organizations may want to consider how to incorporate healthy nudges through text messages to reach rural families with adolescents as a way to improve health outcomes.

Funding: 2016-69001-24915.

\section{NP29 Preliminary Findings From the Healthy Schoolhouse 2.0}

Anastasia Snelling, PhD, RD, American University; Sarah Irvine Belson, PhD, sarah@american.edu, American University, 4801 Massachusetts Avenue, NW, Suite 445, Washington, DC 20016; Erin Watts, MPH;

Melissa Hawkins, PhD; Rachel Albershardt, MS; Gurpreet Kaur, MD

Objective: The goal of Healthy Schoolhouse 2.0 is to improve health literacy and prevent obesity in elementary school students in Washington, DC.

Description: In its first year, the program team has designed and implemented research, education, and extension activities in two elementary schools. Activities in the experimental setting include a professional development (PD) program that help educate teachers on integrating nutrition concepts into the classroom. The PD is focused on the utilization of USDA's Serving Up My Plate: A Yummy Curriculum and has provided teachers with model lessons and instructional kits, as well as fidelity observations of teachers. PD support also includes health promoting programs for teachers, including a pedometer challenge and yoga activities. Extension programming includes working with a local farm-to-table program and coordinating food education programs.

Evaluation: Research is underway in both the experimental and control school, including the collection of assessments of students' nutrition literacy and teachers' self-efficacy and attitudes toward teaching nutrition. The team also has collected plate waste data on students' fruit and vegetable consumption at both sites. Preliminary findings include no significant differences between student demographics and knowledge on the baseline pre-knowledge assessment in the experimental and control settings, suggesting that any growth in the final assessments (to be completed in June 2018) will the attributable to program activities. Pre-program assessments in teacher attitudes at both experimental and control settings are positive, though we may discover a ceiling effect for this measure in both settings. To date, approximately 60 nutrition lessons have been taught. The team has observed differences in the food service in each setting and will explore how the environment effects food consumption.

Conclusions and Implications: Lessons learned include activities to increase lesson implementation as well as programming designed to engage teachers as agents of change by creating a culture of health within their classrooms and in the school.

Funding: 2017-68001-26356. 


\section{NP30 School Dining Redesign: Impacts on Students and Teachers in Middle and High Schools in California}

Lorrene Ritchie, PhD, MS, RD, lritchie@ucanr.edu, University of California, Division of Agriculture and Natural Resources, Nutrition Policy Institute, 2115 Milvia Street, Suite 301, Berkeley, CA 94704; Annie Reed, MPP, University of California, Berkeley, School of Public Health; Kellie Ottoboni, BS; Hannah Thompson, PhD, MPH; Marissa Neelon, MS, RD, UC Cooperative Extension, Contra Costa County; Kris Madsen, MPH, MD, University of California, Berkeley, School of Public Health

Objective: The purpose of the study was to evaluate a school dining redesign in a large school district in California. Outcomes included school lunch participation, student intake of fruits and vegetables, and teacher promotion of school meals.

Description: Using a quasi-experimental controlled trial design, we present results from the first year of a 2-year implementation of a 3-pronged intervention: dining room redesign, distributed points of sale of school lunch, and staff promotion of school meals. Linear regression models were used to compare changes from 2016 to 2017 in survey responses from 7 th-10th grade students and teachers from 12 middle and high schools compared to 12 control schools. Models of student survey data were adjusted for race/ ethnicity, gender and grade. Changes from 2016-2017 in school lunch participation were adjusted for school enrollment, free and reduced price meal enrollment, and student race/ethnicity.

Evaluation: Surveys were collected from over 8,000 students and over 500 teachers. Half of students were Asian, 17\% Latino, 10\% White, 4\% African-American. Significantly more students in intervention compared to control schools reported eating school breakfast and getting lunch from a mobile school cart. More intervention students indicated that they liked the fruit served with lunch, staff encouraged them to eat school lunch, and the school lunch was healthier than foods from elsewhere. Compared to teachers at control schools, teachers at intervention schools reported more often eating the school lunch, and eating from the healthy student vending machines as opposed to the traditional staff vending machines. No increases in the weekly frequency of students eating fruits and vegetables or in teachers promoting school meals to students were detected. Trends in school lunch participation appear promising.

Conclusions and Implications: Preliminary outcome data suggest that a school dining redesign may be effective in improving student perceptions of school meals and participation in school lunch. Future analyses will include 2-year results and assessment of school meal waste.

Funding: 2015-68001-23236.

\section{NP31 Starting Early/Empezando Temprano: Randomized Control Trial to Test the Effectiveness of a Child Obesity Prevention Program}

Mary Jo Messito, MD, mary.messito@nyumc.org, NYU School of Medicine Bellevue Hospital, 462 First Avenue, New York, NY 10016; Alan Mendelsohn;

Roberta Scheinmann, MS; Rachel Gross, MS, MD

Objective: To improve maternal infant feeding and activity practices and reduce child obesity with the Starting Early Program (StEP): a primary care-based obesity prevention intervention beginning in pregnancy for lowincome Hispanic mother-infant dyads.

Description: Conduct a randomized control trial of the StEP intervention. Women enrolled in the third trimester of pregnancy at an urban public hospital clinic, randomized to StEP intervention vs. standard primary care. Intervention included: individual nutrition counseling in prenatal/peri-partum periods, nutrition and parenting support groups at every well child visit with 2 individual and 13 group intervention sessions scheduled prior to child age 3 years. Feeding styles, diet and lifestyle patterns, psychosocial stressors, and demographic information collected at baseline in pregnancy and infant age 3, 10, 19, months, 2 and 3 years, with measured length/height and weight. Multilevel modeling used to assess intervention impact on infant weight for length z-scores (WFLz). T tests and chi square used to assess impact on nutrition knowledge and practices. Logistic regression used to assess intervention dose on risk of obesity (BMI $\geq 95$ th\% for age and sex) at age 3 .

Evaluation: Baseline prenatal assessments $(n=566)$ found high rates of maternal stress, obesity and unhealthy dietary practices. Five hundred and thirty three pregnant women randomized to StEP vs. standard care. No group differences in baseline mother/infant characteristics. Mean sessions attended by 3 years: 8.5 (range 1-15). At infant age 3 months: higher nutrition knowledge, higher rates of exclusive breastfeeding, tummy time and less early introduction of complimentary foods for intervention group. At 10 months, more exclusive breastfeeding, more responsive feeding styles and less introduction of juice. At age 2, more family meals and less restaurant food. Complete weight data available for infants through age 18 months $(n=433)$. Intervention group infants had lower mean WFLz between ages 12-18 months (-.21 (.10), $\mathrm{p}=.046)$. At 36 months, infants attending $>10$ sessions were less likely to have obesity, $\mathrm{p}=.039$.

Conclusions and Implications: Interventions like StEP have the potential to improve maternal infant feeding and activity practices and reduce childhood obesity.

Funding: 2011-68001-30207. 